\title{
The Effect of the Characteristics of Industrial Clusters on Population Urbanization: A Case Study of the Poplar Industrial Cluster in North Jiangsu Province of China
}

\author{
Shiqing Yan \\ College of Urban and Planning, Yancheng Teachers University, Yancheng, China \\ Email: 18360494968@163.com
}

How to cite this paper: Yan, S.Q. (2017) The Effect of the Characteristics of Industrial Clusters on Population Urbanization: A Case Study of the Poplar Industrial Cluster in North Jiangsu Province of China. Open Journal of Social Sciences, 5, 148-168. https://doi.org/10.4236/jss.2017.510014

Received: September 9, 2017

Accepted: October 15, 2017

Published: October 18, 2017

Copyright $\odot 2017$ by author and Scientific Research Publishing Inc. This work is licensed under the Creative Commons Attribution International License (CC BY 4.0).

http://creativecommons.org/licenses/by/4.0/

\begin{abstract}
According to the study on the characteristics of poplar industrial cluster and it's population effect in North Jiangsu, we find that the urbanization transformation of the quantitative characteristics, structural features and flow characteristics of population, and the characteristics of regional settlement are obvious in the process of the poplar industrial cluster development. The spatial indication, the characteristics of industrial structure, enterprise composition and spatial heterogeneity of industrial clusters are the important factors influencing the urbanization of rural population. Cultivating local industrial clusters that are suitable for regional natural, economic and social environment is an important measure to promote the in-situ urbanization of rural population.
\end{abstract}

\section{Keywords}

Characteristics of Industrial Clusters, Rural Population, Local Urbanization, North Jiangsu Province

\section{Introduction}

With the development of industrialization and urbanization, some villages in the eastern coastal areas of China did not show the phenomenon of large-scale population migrate to towns, but showed the nearest and local urbanization in the rural areas [1]. Local urbanization is a phenomenon of urbanization with Chinese characteristics formed under the background of high population density [2]. When the rural economy develop into a certain extent, farmers no longer 
blindly migrate to large and medium cities, and they live in the original residence around the central village or small town, and gradually realize the local non-agricultural employment and local urbanization model [3]. The theoretical study of the local urbanization can be originated from the theory of small town development in the 1980s [4]. Rural labor forces work in the local non-agricultural industry, which makes small towns become the transition zone between urban and rural, and it plays the role of blocking and accumulation of population movement and effectively prevents the population concentrate in the city [5]. There are different ways to define the concepts of local urbanization and the nearest urbanization. From the aspect of space distance, the local urbanization refers to the travel distance of rural residents is between $200 \mathrm{~km}$ to $300 \mathrm{~km}$. From the view of traffic time, the one-way commuting time in the local urbanization is 1 hour to 3 hours, or rural residents can make round trip within one day or make periodic round trip with 3 days to 5 days [6]. From the administrative area, the rural labor forces migrate with the county, and achieve non-agricultural employment and urbanization is known as the local urbanization, while the migration scope across the county or the city and the realization of non-agricultural employment and urbanization is called the nearest urbanization, inter-provincial migration and the realization of non-agricultural employment and urbanization is offsite urbanization [7]. Population local urbanization has a variety of modes, such as the outskirts areas of the metropolitan urbanization, local elites lead to the local urbanization and external resources into the village [2]. Some people think that the local urbanization model has market oriented, policy oriented and people lead these three categories [8]. The driving forces of local urbanization include the transformation of industry cultivation and employment structure, the capitalization of land, the ability of local elite to integrate resources, the impact of traffic mobility and the willingness of farmers to return home [2]. The contrast relationship between rural and urban on labor force rally determines whether rural labors are local urbanization or offsite urbanization [9]. In the local urbanization, the employment model of rural labor force can be divided into three categories: agricultural employment mode, agriculture-industrial employment mode and agriculture-service industry employment mode [6]. In addition, scholars have also studied the positive effects of rural industrial and tourism development on the local urbanization [10] [11] [12] [13]. Researchers studied the concept, mode and power of the local urbanization, which enrich and deepen the theory of the local urbanization. However, the impact of the rural non-agricultural industry on the local urbanization and the impact of industrial characteristics on the local urbanization need further study.

Industrial cluster is a large number of enterprises that have closely linked industries and related support agencies gather together and form the phenomenon of strong and sustained competitive advantage [14]. The theory of industrial cluster is an important theory of regional economic development, which is highly regarded by academics and decision-makers [15] [16]. The development of industrial clusters can promote regional economic growth [17], so that un- 
derdeveloped areas to achieve leapfrog development [18]. The development of industrial clusters can accelerate the construction of regional innovation systems [19], and enhance regional innovation capacity [20] and regional competitiveness [21]. Industrial clusters with the function of talent development, is conducive to the accumulation of high-level talents [22], to guide the transformation of personnel training mode of higher education [23]. There is a close relationship between the development of industrial clusters and regional culture. The cultivation and development of culture make the progress and development of the main actors have strong persistence [24], and then have positive influences on the development of industrial clusters. Recent studies have found that the development of industrial clusters have a specific population effect, the development of industrial clusters can change the characteristics of regional population flow and population structure characteristics [25]. The healthy development of specific industrial clusters also has a positive effect on avoiding regional endowment risks [26].

Due to industrial clusters gather a large number of interconnected companies and institutions, they absorb a certain amount of labor, and then play an important role in promoting regional population urbanization. Different characteristics of industrial clusters have different influence on urbanization. However, many researchers neglected the impact of the characteristics of industrial clusters on population urbanization. Investigating the characteristics of industrial clusters and the effect of the characteristics of industrial clusters on population urbanization, and analyzing the effect formation mechanism of regional population urbanization caused by the development of the poplar industrial cluster can enrich the theory of industrial clusters, expand the application scope of industrial cluster theory, reveal the dynamic mechanism of urbanization of rural population, and provide theoretical guidance to formulate scientific and rational strategy of population urbanization.

This paper examines the process of the poplar industry cluster development, analyzes the characteristics of the poplar industrial cluster in North Jiangsu and the effects of the characteristics of poplar industrial cluster on population urbanization, and finally reveals the influence mechanism of industrial cluster characteristics on the local urbanization of rural population.

\section{The Development of Poplar Industrial Cluster in North Jiangsu}

The poplar industrial cluster in North Jiangsu refers to a series of industries that developed from the production and processing of poplar wood including the poplar nursery, poplar forest construction, pruning, pest control; to poplar veneer, production and trade of wood products; production, sales and maintenance of the machine for wood processing; production and sale of auxiliary materials; training and consulting of the technology of poplar production and processing; enterprises human resources management and services, legal services and so on. 
The poplar industry clusters in North Jiangsu originated from the poplar cultivation in Siyang County. In the early 1970s, the original Nanjing Forestry College introduced the southern type of poplar seedlings and grew successfully in Siyang County at the first time. In the 1980s, the fall of grain prices leaded to the adjustment of the industrial structure in the northern Jiangsu Province which are the main grain-producing areas and expanded the cultivation of poplar in the "four-side land", and then the scale of poplar cultivation continued to expand. In the late 1990s, in order to speed up the pace of industrialization, a number of counties and cities in North Jiangsu expand poplar cultivation area, and making this region become the largest poplar concentrated distribution area in China.

With the expansion of poplar cultivation, the poplar wood processing industry developed and the poplar industrial cluster formed and continuously developed in North Jiangsu. In 1978, the original Daxing brigade in Linhe commune Siyang County began to use poplar wood processing board and product the plywood. After the 1980s, poplar cultivation area has been expanding, and the implementation of household contract responsibility system in rural areas, the small micro-enterprises which used poplar to product plywood gradually increased, expanding the production scale of poplar sheet. In the late 1990s, under the active encouragement and support of the government, a number of wood processing area which use poplar wood as raw material emerged in northern Jiangsu. Therefore, poplar cultivation, pruning, pest control, poplar processing, plywood production, furniture production and design, poplar cultivation and poplar processing training, financing, etc. emerged in northern Jiangsu.

\section{The Characteristics of the Poplar Industrial Cluster in North Jiangsu}

Poplar industrial cluster in northern Jiangsu was developed on the basis of poplar cultivation and then gradually develop to the poplar processing, wood processing machinery manufacturing, and the transportation of timber and its products. In some respects such as the spatial indication, the characteristics of industrial structure, enterprise composition and spatial heterogeneity, the poplar industrial clusters have distinctive features which different from other industrial clusters.

\subsection{The Poplar Industry Cluster in North Jiangsu Embedded in the Rural Area}

The root ability of industrial clusters refers to industrial clusters are rooted in the specific natural conditions, economic and (or) socio-cultural conditions. If taking into account the spatial differences of natural, economic, social and cultural conditions, thus the root characteristics of the industrial cluster have spatial characteristics, namely spatial directivity. Directing to a specific area is one of the important characteristics of poplar industrial cluster in northern Jiangsu. 
The formation of the poplar industry cluster benefit from the specific natural conditions in North Jiangsu. The southern type of poplar seedlings introduced by the original Nanjing Forestry College in the early 1970s, come from the southern provenance of the black poplar variant ( $P$. deltoids Bartr. Var. deltoids) in the alluvial plain of Mississippi River to the south of $35^{\circ} \mathrm{N}$ in North America, where is humid subtropical monsoon climate, While Siyang county is located in the transitional zone of subtropical monsoon climate to warm temperate monsoon climate. The water and heat conditions of these two areas are very close to each other, so North Jiangsu is a good area to grow the poplar species. From the soil conditions, most parts of North Jiangsu is the flood plain of the Yellow River, where has a large area of sands and loam that have loose structure and deep soil, it is one of the most advantageous region for poplar growth. As the natural conditions are superior, the poplar species grow fast and have good material at North Jiangsu.

The formation of poplar industry clusters adapt to the local economy. The level of economic development of North Jiangsu is low, and the proportion of primary industry is large, which provides opportunity for the development of poplar industrial cluster. One hand, since 1978 the decline in grain prices has exerted a major impact on north Jiangsu where crop cultivation is the mainstay, stimulating local farmers to build poplar shelterbelt and to use the wastelands to cultivate poplar, which expand continuously the poplar cultivation area. On the other hand, the degree of industrialization in north Jiangsu is low, and the poplar processing industrialization based on the cultivation of poplar is an important method to promote the industrialization process. In 1996, it was separated from Huai'an City and upgraded to the provincial municipality, named as Suqian City, which was the city with the highest enthusiasm to develop the poplar industry. After 1999, Suqian City adjusted the structure of the agricultural industry and focused on the development of poplar industry to accelerate the industrialization process. Since 2000, the government of Suqian City carried out activities to encourage farmers to plant poplar very year. Besides, 2001 was also identified as the "Poplar Industry Year" to promote the development of poplar industry.

Poplar wood processing industry should be located in the raw materials areas. Poplar logs as the raw materials of poplar wood processing industry which is resource-intensive industries and need to consume large amounts of wood. While poplar logs are loss heavily raw materials, about $5 \%-8 \%$ of the quality of the bark, scrap and broken veneer etc. cannot be transferred to the finished plate during the process of wood processing. Furthermore, logs that are used to product veneers often contain water and the quality of veneer products has decreased after air-dried. Therefore, poplar wood processing industry layout in the rural of North Jiangsu with rich poplar wood will have a higher economic efficiency.

Poplar processing industry should be located in rural areas with low land prices. Take veneer production as an example, veneer production enterprises occupy more land area. When a veneer production enterprise has a wood peel- 
ing machine with production capacity $2000 \mathrm{~m}^{3} / \mathrm{a}$ and employ three labors, it approximately needs production land $2000 \mathrm{~m}^{2}, \log$ yard $1000 \mathrm{~m}^{2}$, veneer drying site $1700 \mathrm{~m}^{2}$, and some other non-productive sites such as workers resting places, thus the whole enterprise covers an area about $0.5 \mathrm{hm}^{2}$. If considering rainy weather may prolong veneer drying time, the whole enterprise needs land about $0.7 \mathrm{hm}^{2}$. Besides, the scale of wood veneer production enterprises should not be too large. Since veneer slabs that peeled from the poplar logs need to be transported to the air-dried sites to be fixed and dried in the veneer production process, thus the larger the scale of production enterprises, the farther the transportation distance and the higher the damage rate of veneer slabs. If the scale of veneer slabs production enterprises over the best production scale will inevitably reduce production efficiency. Therefore, veneer production enterprises must choose to locate in the rural areas with low land prices.

In summary, the wood processing industry layout in rural areas of North Jiangsu where is the poplar origin place, due to industry association, information and infrastructure sharing, regional branding and some other reasons, the poplar industrial cluster in North Jiangsu has the spatial indication that clearly concentrated on rural areas of north Jiangsu.

\subsection{The Poplar Industry Cluster in North Jiangsu Is Mainly Made Up of Labor Intensive Industry}

With the expansion of poplar cultivation area and the development of poplar processing industry in northern Jiangsu, the industry continues to differentiate. Poplar cultivation industry differentiates into different industries, such as some people are specializing in nursery cultivation of poplar seedlings and others who are specializing in poplar cutting. In the context of the outflow of rural labor, some people are devoted to prune poplar branches and control poplar pests in the slack season. The development of poplar processing industry in North Jiangsu leads to the development of the tertiary industry, such as purchase and sale of poplar wood; manufacturing, sale and maintenance of wood processing machinery and accessories; transportation and sale of scrap and auxiliary materials in the wood processing industry. And both these industries are labor-intensive industries (see Table 1).

In recent years, the poplar industry cluster in northern Jiangsu differentiates the consultation of poplar nursery, cultivation, pest control, and the technical training industry of timber processing, Such as Nanjing Forestry University, National Academy of Forestry and other institutions of higher learning and scientific research institutes come to Siyang County and do technical training and business guidance every year. In addition, some places have law firms, investment and financing institutions to provide specifically services for the local timber processing enterprises. But these industrial sectors just occupied for a small proportion in the whole industry cluster, thus the entire poplar industry clusters are still mainly composed of labor-intensive industries. 
Table 1. Industrial structure of the poplar industrial cluster in the North Jiangsu.

\begin{tabular}{ll}
\hline Industry Category & \multicolumn{1}{c}{ Specific industry sector } \\
\hline Primary industry & $\begin{array}{l}\text { Poplar nursery, cultivation, pest and disease control, pruning branches, cut } \\
\text { down the poplar }\end{array}$ \\
Secondary industry & $\begin{array}{l}\text { Manufacture of wood panels, sheet materials, finished boards, furniture } \\
\text { hardware, furniture and wood processing equipment, etc. }\end{array}$ \\
& $\begin{array}{l}\text { Purchase and sale of poplar and its scrap, sales and maintenance of wood } \\
\text { processing equipment, recruitment of employees in related enterprises, legal } \\
\text { services and financial services, technical consultation and training in related } \\
\text { fields }\end{array}$ \\
\hline
\end{tabular}

Data source: Author's survey. At the end of 2014, the author surveyed the five wood processing areas, Xichenji Town - Yanghe Town in Sucheng District, Suqian City; Zhongxin Town - Linhe Town in Siyang County, Suqian City; Xianguan Town - Sangxu Town in Shuyang County.

\subsection{The Poplar Industrial Cluster in North Jiangsu Is Mainly Composed of Small Enterprises}

Most of poplar industries in northern Jiangsu are small and micro enterprises, the poplar nursery and cultivation in the primary industry are main in single-family operation, and the production scale are small; pruning of poplar branches, pest control, cutting down the poplar are also small enterprises and most of enterprises just have 3 - 5 employees. The enterprises size of production of wood products and wood processing equipment manufacturing in the second industry are large, but the number of these enterprises is very small. However, the wood coil enterprises tend to be micro-enterprises with 3 - 10 employees, and the number of enterprises is huge. Survey has shown that the Daxing community in Siyang County Wood Park has more than 700 enterprises and there are more than 600 wood coil businesses. The transportation of timber, its waste and timber products, sales and maintenance of timber processing equipment, corporate human resources services, technical advice and personnel training, legal and financial services in the third industry are all small and micro enterprises. Therefore, in poplar industrial cluster in North Jiangsu, only a small number of large and medium-sized enterprises in the secondary industry, small and micro enterprises accounting for the absolute superiority in the number and employment.

\subsection{The Primary Industry of Poplar Industrial Cluster Distributed as a Surface, the Secondary and Tertiary Industries Concentrated in Several Points}

The primary industry of poplar industrial cluster in North Jiangsu is in a planar distribution. At early ages, poplar cultivation industry in Siyang County was planar distribution, and continues to expand to the surrounding areas. Nowadays, the poplar cultivation industry throughout the five jurisdiction cities under Jingsu province in the north (Figure 1), and expanding to central Jiangsu, northern Anhui, eastern He'nan and southern Shandong etc.

The secondary and tertiary industries of poplar industrial cluster in North Jiangsu are characterized by point-like distribution. These five poplar processing 


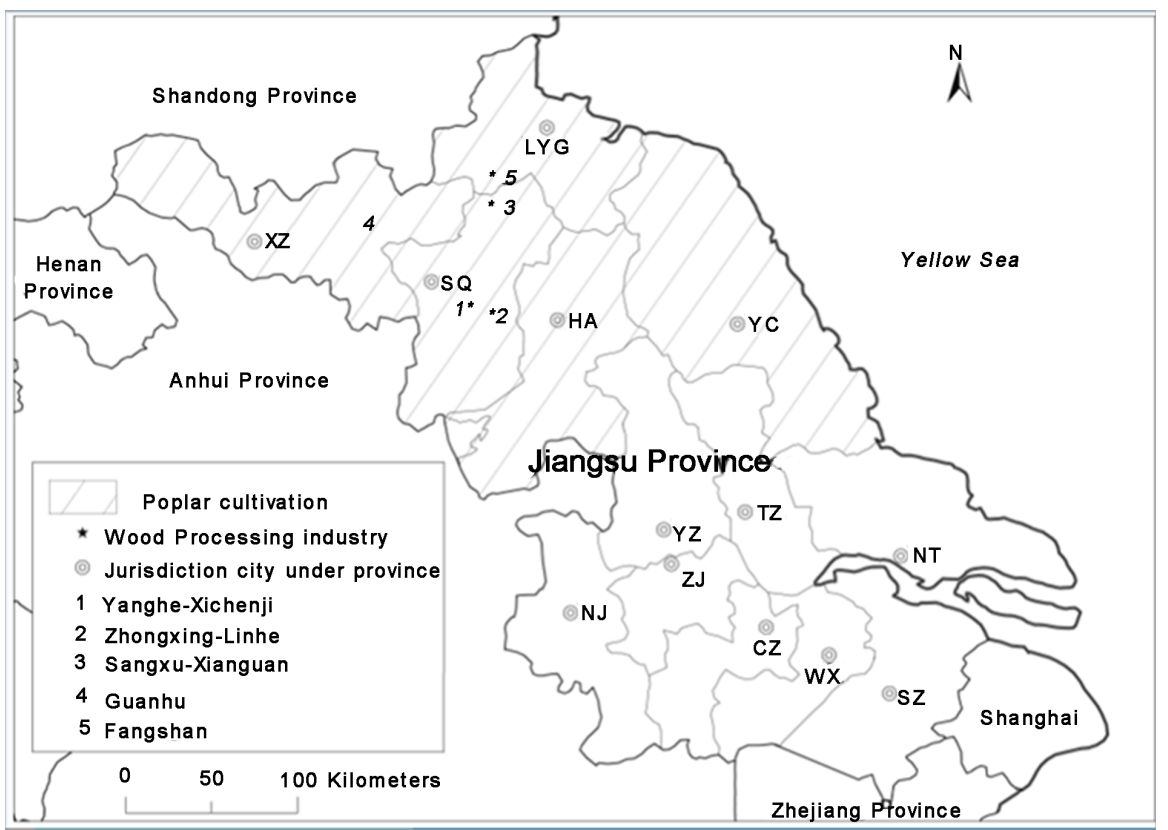

Figure 1. Distribution of the industries in poplar industry cluster in North Jiangsu.

areas: Yanghe Town to XiChenji Town in Yanghe District of Suqian City, Zhongxing Town to Lin-he town in Siyang County, Sangxu Town to Xianguan Town in Shuyang County, Guanhu town in Pizhou City, Fangshan Town in Donghai County are the concentrated distribution points of second industries like poplar processing and the associated tertiary industries (Figure 1).

From the perspective of industrial linkages, the primary, the secondary and the tertiary industries of poplar industrial cluster in North Jiangsu combine closely and promote each other. The poplar cultivation industry which is in the form of planar distribution provide raw materials to poplar wood processing industry and become the prerequisite and foundation for the development of the secondary industry. The emergence and development of the poplar processing industry with point form distribution provides markets for the poplar cultivation and make it to be commodity production, which promotes the expansion of the spatial scope of the poplar cultivation. The poplar cultivation and the poplar processing industry provide conditions for the development of related tertiary industry and lead to the distribution of the tertiary industry is point distribution. Thus poplar industrial cluster should include point-like poplar processing areas and plane-like poplar cultivation area.

From the perspective of industrial spatial distribution, the consistence of intra-regional and the difference of inter-regional are obvious. The economic development level and the characteristics of industrial structure of the northern Jiangsu have similarities; the production preference of people and the policy system of government have commonality; language and culture are similar. But there are obvious differences in economic, social, cultural and policy among northern Jiangsu, central Jiangsu, northern Anhui, eastern Henan and southern Shandong. 
Based on the above considerations, the boundary of poplar industrial cluster in North Jiangsu is defined as the administrative scope of northern Jiangsu province. And the area of poplar cultivation industry in planar distribution is the coverage region of poplar industry cluster, and the five point-like poplar processing areas are the cores of the poplar industrial cluster.

\section{The Effect of the Poplar Industrial Cluster on the Urbanization in North Jiangsu}

In the development of poplar industrial cluster in North Jiangsu, wood processing areas have created a lot of jobs, so they have the function to attract the people who live in nearby to gather in these points. On the basis of population agglomeration, the population structure and living characteristics of the timber processing area are unique.

\subsection{The Changes of the Characteristics of Regional Population Flow: Wood Processing Areas Became Centers to Attract Regional Population}

In the development of poplar industrial cluster in North Jiangsu, wood processing areas attract a number of young and middle-aged laborers, and some young laborers and their dependents settle down, so these areas are population moving centers. Among them, Daxing community in the Siyang County Wood Park, Sangxu Town - Xianguan Town in Shuyang County, Guanhu Town in Pizhou City, Fangshan Town in Donghai County are new population attraction centers that formed by poplar industrial cluster development. The Yanghe Town-Xi Chenji Town is a traditional population attracting center, for it is close to the Yanghe Town the famous wine-making center. The traditional wine industry has a certain attraction to the outside population, and the development of poplar industrial cluster in the area strengthens the population attraction center of the district. The formation of new population attraction centers or the strengthening of the original population attraction centers will inevitably be accompanied by changes of the regional population flow characteristics, regional population number characteristics and regional settlement.

\subsubsection{Wood Processing Areas Become the Daytime Population Gathering Center}

The young and middle-aged labors who are attracted to wood processing areas, they work there during the day time and go back home at night, which result in a change of the ratio of day population to night population in wood processing areas and a change of the "one-day tide" characteristic of the population flow field.

The ratio of daytime population to night population increases. Typically, labors in suburban rural communities who migrate to work during the day time and go home to rest at night, the ratio of day and night population is less than 1; young and middle-aged migrant workers in rural communities tend to settle in places of employment, and the ratio of day to night population is about 1. Due to 
the development of poplar industrial clusters, the five wood processing areas attract the surrounding labors to work here during the day time and come home at night, so the ration of day and night population is greater than 1 . By the end of 2014, the continuous survey in Daxing community of Siyang County Wood Park found that the ratio of the number of day and night population in continuous five days are between 1.5 - 1.6, which is higher than in Siyang County suburbs and rural communities.

The characteristic of "one-day tide" of the population flow has changed. The ratio of day to night population is closely related to the "one-day tide" phenomenon in the population flow. Usually, there is a "tidal wave system" between the city (town) and the surrounding villages in a one-day cycle of population movement. A part of the labors in villages are attracted to work in urban during the daytime and go back to villages at night, so it leads the formation of the "one-day tide" phenomena, that is the "high tide" in the morning and "low tide" in the evening in city or town, while form the "low tide" in the morning and "high tide" in the evening in villages. The suburbs of Siyang county the underdeveloped areas in Jiangsu province, are affected by the population effect of poplar industry cluster, and the "one-day tide" in the population flow are changing. The development of poplar industry cluster has different effects on the "one day tide" phenomenon in the regional population flow. Due to XiChengji town-Yanghe town wood processing area is close to the formerly population attraction center Yanghe town, the development of poplar industrial cluster has increased the strength of the "one-day tide" phenomenon between Yanghe town and the surrounding rural areas. The three wood processing areas: Sangxu town - Xianguan town in Shuyang county, Guanhu Town in Pizhou city and Fangshan town in Donghai county areas located in rural areas and far from the city, and did not have any industries to attract foreign population, so they did not have the "one-day tide" phenomena of population flow before the forming of the poplar industry cluster. However, with the development of polar industrial cluster, there formed the "one-day tide" phenomena of population flow that take these three points as center. The Daxing community of Siyang County Wood Park is suburban is close to Zhngxing town, the development of poplar industrial clusters suppresses the "one-day tide" phenomenon of population flow between Daxing community and Siyang rural areas, replaced the form a "one-day tide" phenomenon of population movement between the Zhongxing town and the Daxing community. Survey shows that the "one-day tide" between the Daxing community and Siyang rural areas only spread to $5 \mathrm{~km}$ around the community.

\subsubsection{Wood Processing Wood Processing Areas Become Relocation Centers for Foreign Population}

The formation or strengthening of the population relocation center makes the population of the wood processing area separate from other rural areas. On the one hand, the increasing of the number of resident population in the wood 
processing area is in distinct contrast to the resident population in other rural areas. In the rapid urbanization, the national rural population continues to decrease. However, the development of poplar industrial clusters in northern Jiangsu has led to an increase in the number of permanent residents in several wood processing areas. Taking Daxing community of Siyang county wood industry park as an example, in 1982 the third census, household population and resident population of the 16 villagers group within the community are equal, both were 5345 people; in 1990 the fourth census, the household population was 7029 people and the resident population was 7852 people; in 2000 the fifth census, the household population and the resident population increased to 8927 people and 9860 people, respectively; in 2010 the sixth census, the household population and the resident population were 9893 and 13,378, respectively. The survey found that in recent years the commercial housing owners in Daxing village generally reflected living in this area brings a lot of convenience to work, shopping and education of children. By the end of 2014, there are 316 migrant workers purchased houses and settled in Daxing village.

On the other hand, the "seasonal tide" phenomenon in the population flow is different from that of other rural areas. Under normal circumstances, some rural labor forces of the less developed areas move to work in the developed cities (towns) after the spring festival and the farming busy seasons, and they go back home to reunite with their family and do short-term farming work during the Spring Festival and farming busy season. Thus, in the developed cities (towns), the population flow forms the "high tide" phenomenon after festival and farming busy season, and the "low tide" phenomenon before festival and farming busy season. However, in the less developed rural, the population flow forms the "high tide" phenomenon before festival and farming busy season, and the "low tide" phenomenon after festival and farming busy season. That is, the "seasonal tide" phenomenon of population movement between underdeveloped rural areas and developed cities (towns). The five wood processing areas of the poplar industry cluster in northern Jiangsu are located in underdeveloped rural areas, the development of poplar industrial clusters has suppressed the "seasonal tide" phenomenon of population movement between the five wood processing areas and the developed cities (towns). Taking Daxing community of Siyang County Wood Industry Park as an example, the development of poplar industry cluster create a large number of jobs for the local, young and middle-aged labor force in the community are almost all work in the local, and the number of migrant workers in the household population less than $1 \%$ of all the labor forces. Therefore, the development of poplar industrial cluster in North Jiangsu effectively "shields" the "seasonal tide" phenomenon of population movement between the wood processing areas and the developed cities (towns).

Therefore, the development of the five wood processing areas, such as Daxing community of Siyang County Wood Industry Park, forming a new population attract centers or strengthening the original population attract center in North 
Jiangsu, and it provides important conditions to localization of urbanization for the rural areas in the area.

\subsection{The Formation of Spatial Differences of Regional Population Structure: The Population Structures of Wood Processing Areas Are Different from the Ordinary Villages}

The rapid advancement of population urbanization, the young and middle-aged labor forces and their dependents gather to the five wood processing areas, thus the population grows rapidly, and the regional population structures change quickly.

\subsubsection{The Age Structure of the Resident Population Is Different from That of Ordinary Village}

Due to the wood processing areas in the poplar industrial clusters attract a large number of rural young and middle-aged labor forces, making the fraction of young and middle-aged populations increase and the proportion of children and juvenile populations decrease. Taking Daxing community of Siyang county wood industry park as an example, "Six general" data show that the community resident population was 13,378 people, of which the population between 0 and 14 years old was 2168 people, accounting for $16.2 \%$; the population between 15 and 59 years old was 538,881 people, accounting for $64.8 \%$; the population elder than 60 years old was 121,398 people, accounting for $14.6 \%$. At the same time, in the resident population of village in the Siyang County, the population between 0 and 14 years old was accounting for $22.6 \%$; the population between 15 and 59 years old was accounting for $61.1 \%$; the population elder than 60 years old was accounting for $16.3 \%$. In the resident population of the whole Siyang County, the population between 0 and 14 years old was accounting for $20.6 \%$; the population between 15 and 59 years old was accounting for $64.8 \%$; the population elder than 60 years old was accounting for $14.6 \%$. In the resident population of village in the Suqian City, the population between 0 and 14 years old was accounting for $20.6 \%$; the population between 15 and 59 years old was accounting for $63.7 \%$; the population elder than 60 years old was accounting for $15.7 \%$. In the resident population of the whole Suqian City, the population between 0 and 14 years old was accounting for $19.0 \%$; the population between 15 and 59 years old was accounting for $67.2 \%$; the population elder than 60 years old was accounting for $13.9 \%$ (see Table 2). Therefore, the development of the poplar industrial cluster in northern Jiangsu leads to the population structure of wood processing area is different from the other rural areas in northern Jiangsu, but it is similar to the city or town.

\subsubsection{The Gender Structure of the Resident Population Is Different from That of Ordinary Village}

In the foreign labor forces that are attracted to wood processing areas in the poplar industrial clusters, the male population is greater than female population, so that the sex ratio of the young and middle-aged population increased higher 
Table 2. The age structure of the resident in Daxing village and that in the areas of comparisonin 2010 .

\begin{tabular}{|c|c|c|c|c|c|c|c|}
\hline \multirow{2}{*}{ Areas } & \multirow{2}{*}{$\begin{array}{l}\text { The total } \\
\text { number of } \\
\text { population }\end{array}$} & \multicolumn{2}{|c|}{0 - 14 years old } & \multicolumn{2}{|c|}{15 - 59 years old } & \multicolumn{2}{|c|}{60 years old and above } \\
\hline & & Number & Ratio & Number & Ratio & Number & Ratio \\
\hline Daxing Village & 13,378 & 2168 & $16.2 \%$ & 10,115 & $75.6 \%$ & 1095 & $8.2 \%$ \\
\hline $\begin{array}{l}\text { The village of } \\
\text { Siyang County }\end{array}$ & 443,212 & 100,032 & $22.6 \%$ & 270,987 & $61.1 \%$ & 72,193 & $16.3 \%$ \\
\hline Siyang County & 831,112 & 170,833 & $20.6 \%$ & 538,881 & $64.8 \%$ & 121,398 & $14.6 \%$ \\
\hline $\begin{array}{l}\text { The village of } \\
\text { Suqian City }\end{array}$ & $2,440,366$ & 501,765 & $20.6 \%$ & $1,555,427$ & $63.7 \%$ & 383,174 & $15.7 \%$ \\
\hline Suqian City & $4,719,178$ & 895,579 & $19.0 \%$ & $3,169,265$ & $67.2 \%$ & 654,334 & $13.9 \%$ \\
\hline
\end{tabular}

Data source: Daxing Village data provided by Siyang County Zhongxing Town Statistics Station, other data from Suqian City 2010 census data compilation.

than that of the ordinary village. Taking Daxing community of Siyang county wood industry park as an example, in 2010 the sixth census, the sex ratio of population between 15 to 59 years old in Daxing Village is 120.9, at the same time, the sex ratio of residential population between 15 to 59 years old in the villages of Siyang County, Siyang County, the villages of Suqian City and Suqian City are 95.4, 98.5, 91.2 and 97.9, respectively. Obviously, the sex ratio of residential population between 15 to 59 years old in Daxing Village is highly larger than other four areas (see Table 3).

From Table 3, we can draw that the gender structure of the children and the aging group of Daxing Village are similar to that of the city. In 2010 the sixth census, the sex ratio of population between 0 to 14 years old of Daxing Village is 126.8 , and the sex ratio of residential population between 0 to 14 years old in the villages of Siyang County, Siyang County, the villages of Suqian City and Suqian City are 127.8, 132.9, 125.3 and 126.8, respectively. The sex ratio of population between 0 to 14 years old in Daxing Village is close to other four areas. Besides, the sex ratio of population of 60 years old and above in Daxing Village is also close to other four areas (see Table 3). According to the above analysis, we get that the development of poplar industrial clusters in northern Jiangsu makes the gender structure of resident population between 15 to 59 years old in wood processing area different from that of surrounding rural areas, but it is similar to urban areas in developed areas.

\subsubsection{The Employment Structure of the Resident Population Is Different from That of Ordinary Village}

The employment structure of the resident population of Daxing Village is clearly different from that of the underdeveloped rural areas. In the background of the rural labor forces flow to the city and the rural resident population is mainly engaged in agricultural production, the development of poplar industry cluster leads to resident population of Daxing Village are mainly employed in the second and third industry. The survey by the end of 2014 shows that there are 
Table 3. The gender structure of the resident population in DaxingVillage and that in the areas comparison in 2010 .

\begin{tabular}{|c|c|c|c|c|c|c|c|c|c|}
\hline \multirow{2}{*}{ Areas } & \multicolumn{3}{|c|}{0 - 14 years old } & \multicolumn{3}{|c|}{15 - 59 years old } & \multicolumn{3}{|c|}{60 years old and above } \\
\hline & Male & Female & $\begin{array}{l}\text { Sex } \\
\text { ratio }\end{array}$ & Male & Female & $\begin{array}{l}\text { Sex } \\
\text { ratio }\end{array}$ & Male & Female & $\begin{array}{l}\text { Sex } \\
\text { ratio }\end{array}$ \\
\hline Daxing Village & 1212 & 956 & 126.8 & 5537 & 4578 & 120.9 & 524 & 571 & 91.8 \\
\hline $\begin{array}{l}\text { The village of } \\
\text { Siyang County }\end{array}$ & 562,99 & 43,733 & 128.7 & 132,335 & 138652 & 95.4 & 34375 & 37,854 & 90.8 \\
\hline Siyang County & 97,471 & 73,362 & 132.9 & 267,401 & 271,480 & 98.5 & 58,347 & 63,051 & 92.5 \\
\hline $\begin{array}{l}\text { The village of } \\
\text { Suqian City }\end{array}$ & 279,017 & 222,748 & 125.3 & 762,567 & 792,860 & 91.2 & $1,81,686$ & 201,488 & 90.2 \\
\hline Suqian City & 500,658 & 394,921 & 126.8 & $1,567,760$ & $1,601,505$ & 97.9 & 311,939 & 342,395 & 91.1 \\
\hline
\end{tabular}

Data source: Daxing Village data provided by Siyang County Zhongxing Town Statistics Station, other data from Suqian City 2010 census data compilation

about 3400 resident populations from all workers of the wood processing enterprises of Daxing Village, the resident population accounts for about half from nearly 1000 people who are engaged in wood trade and transportation, and the population who worked in the commercial shops, hotels, restaurants and other third industry up to 800 people ${ }^{1}$. This is a significant difference of employment structure between the Daxing Village and the surrounding rural areas in which majority of resident population are employed in the primary industry. In addition, Daxing Village also divided new occupation that the surrounding rural areas do not have, such as manager of large and medium-sized enterprise, and brokers of wood and its products. The change of employment structure caused by the development of poplar industry cluster is one of the most important characteristics of population urbanization.

\subsubsection{The Household Registration Structure of the Resident Population Is Different from That of Ordinary Village}

The survey found that the four wood processing areas attract a large number of foreign labors, leading to "separation of people and household" phenomenon that ordinary villages do not ha, "Six general" data show that resident population of Daxing Village is 13,378 ve. Taking Daxing Village of Siyang County wood industry park as an example epeople, of which the number of people who are separate with their household is 3485 , and the fraction of people who are separate with their household is $26.1 \%$. At the same time, the fraction of people who are separate with their household of the Zhongxing Town, Siyang County, Suqian urban area and Suqian City are 11.5\%, 5.6\%, 12.1\% and 9.7\%, respectively (see Table 4).

\footnotetext{
${ }^{1}$ At the end of 2014, the author surveyed the five wood processing areas, Xichenji town - Yanghe town in Sucheng District, Suqian city; Zhongxin town - Linhe town in Siyang county, Suqian city; Xianguan town - Sangxut own in Shuyang county, Suqian city; Fangshan town in Donghai county, Lianyungang city and Guanhu town in Pizhou City. Bingquan Lin who works at Suqian Planning Bureau and Di Zuo who is a graduate student of Tianjing Normal University participate this survey.
} 
Table 4. The Phenomenon of "separation of people and their household" in Daxing village and the areas surround.

\begin{tabular}{cccc}
\hline Areas & $\begin{array}{c}\text { The number of } \\
\text { residential } \\
\text { population }\end{array}$ & $\begin{array}{c}\text { The number of people } \\
\text { who are separate with } \\
\text { their household }\end{array}$ & $\begin{array}{c}\text { The ratio of people who } \\
\text { are separate with their } \\
\text { household }\end{array}$ \\
\hline Daxing Village & 13,378 & 3485 & $26.1 \%$ \\
Zhongxing Town & 273,152 & 31,321 & $11.5 \%$ \\
Siyang County & 831,112 & 46,911 & $5.6 \%$ \\
Suqian urban area & $1,437,685$ & 173,812 & $12.1 \%$ \\
Suqian City & $4,719,178$ & 458,603 & $9.7 \%$ \\
\hline
\end{tabular}

Data source: Daxing Village data provided by Siyang County Zhongxing Town Statistics Station, other data from Suqian City 2010 census data compilation.

\subsubsection{The Other Social Structures of the Resident Population Are Different from That of Ordinary Village}

Further, our survey also finds that the family structure of the resident population of these four wood processing areas is different from the surrounding rural areas, the proportion of families separated by generation, empty-nest families, left-behind women and children of these four wood processing areas are much lower than that of neighboring villages. The enrollment of large and medium-sized enterprises has increased the education period of the staff and workers, so that the education period of resident population of Daxing Village is significantly higher than the surrounding villages, some research and development institutions have a few master degree and doctoral degree owners, engineers and senior engineers and other high title staffs. Although the number is small, the surrounding villages and ordinary rural areas have not owned highly educated talents.

Therefore, the social structures of the resident population of these four wood processing areas are different from that of surrounding rural areas, but they are similar to the social structures of urban areas.

\subsection{The Formation of Spatial Difference of Regional Settlement: The Settlement of the Wood Processing Areas Is Different from That of Ordinary Village}

In the context of rapid urbanization, the population who live in underdeveloped ordinary rural areas is declining and the hollowness is increasing. Although rural settlements occasionally gather in convenient locations, due to the lack of industry support or service facilities, resulting in the gathering process is very short, and ultimately can not form the settlements with a certain population size and the corresponding economic and social functions. The development of poplar industrial clusters in northern Jiangsu lead to population concentrate in the wood processing areas, and the original rural settlements gradually evolved into town settlements with specific economic and social functions and these town settlements have centralized living areas, gathered a certain size of the secondary 
and tertiary industries, infrastructure and service facilities.

Survey shows that the population living space of the Daxing Village in Siyang County Wood Industry Park obviously present "polarization" phenomenon. In recent years, the speed of residents of Daxing Village move to live in the regions near the intersection of Xuhuai Road and Linzheng Road is rapidly increasing. In particular, since the Siyang County Wood Industry Park established in the Daxing Village in 2000, the number of population who relocate to the intersection of the two roads grew faster. On August 2008, the new rural concentrated residence that covers 110 acres was built in the northeast corner of the intersection of thetwo roads, and 385 households have all been admitted. On October 2011, Yiyang apartment that covers 96 acres was built in the northwest side of the intersection, and the current number of households has been more than 600 . At the end of 2013, the Daxing Riverside Garden which located in the north side of the intersection was built, the residential area covers 80 acres, there are housing 800 sets, and the occupancy rate has more than 50\%. The Daxing Times Square on the south side of intersection will be completed, and in the future the apartments that cover $30,000 \mathrm{~m}^{2}$ will attract many residents to live. Commercial houses rapidly gather in the intersection of the two roads, which leads to the living space of this village shown as clustered trend.

In summary, the development of poplar industrial clusters in North Jiangsu is an important impetus to urbanization of rural population. The five wood processing areas in the cluster are located in the hinterland of northern Jiangsu, the development of the wood processing industry and related industries have made the wood processing areas gradually becoming a regional population attraction center, the employment structure of the residents is obviously changed, the structural characteristics of the resident population are obviously different from those of the ordinary rural areas, and the settlements are gradually evolved from ordinary villages into town communities that integrate with residential, industrial and commercial. These changes indicate that the wood processing areas in the poplar industrial cluster in North Jiangsu are no longer traditional villages, but they are gradually transformed into towns.

\section{The Influence Mechanism of Poplar Industrial Cluster Characteristics on Local Urbanization in Northern Jiangsu}

Poplar industrial clusters in northern Jiangsu have specific characteristics in spatial indication, industrial structure, enterprise structure and spatial differentiation. These characteristics have important impacts on regional population urbanization.

\subsection{The Influence Mechanism of the Spatial Indication of Poplar Industrial Cluster on Local Urbanization}

The poplar industrial cluster in northern Jiangsu is rooted in the rural areas of northern Jiangsu, and its spatial indication has a positive impact on the rural population local urbanization. The spatial indication characteristic of poplar in- 
dustrial clusters in northern Jiangsu determines the wood processing industry tends to develop in northern Jiangsu rural areas, the labor forces attracted by industrial cluster achieve local urbanization in the rural hinterland of northern Jiangsu. As the cost of living in rural areas of northern Jiangsu is much lower than that in developed areas, and then accompanied by the migration phenomenon of the dependents of young and middle-aged labor forces absorbed by the poplar industrial clusters in northern Jiangsu, which makes the proportion of migrations increase and benefits the improvement of the urbanization level. The high employment rate of young and middle-aged workers and the high immigration rate of their dependents have become an important driving force for the evolution of rural settlements into town settlements.

\subsection{The Influence Mechanism of Industrial Structure Characteristics of Polar Industrial Clusters on Local Urbanization}

The poplar industrial cluster in northern Jiangsu is mainly composed of labor-intensive industrial sector, which is conducive to population urbanization. On the one hand, the development of industrial clusters requires a lot of young and middle-aged labor force, while rural population is dense and labor resources are rich in northern Jiangsu. The industrial structure characteristics of the polar industrial cluster is consistent with the regional labor force structural characteristics, attracting a large number of young male laborers to work in this areas, and promoting the progress of the local urbanization. On the other hand, young and middle-aged labor forces work in local area lead to the age structure, gender structure, academic structure, occupation structure of population are different from the surrounding villages. Therefore, the above two reasons make the characteristics of population flow and population structure in the specific area of poplar industry cluster are different from ordinary rural areas, and they are also different from that of large and medium cities.

\subsection{The Influence Mechanism of Enterprise Composition Characteristics of Polar Industrial Clusters on Local Urbanization}

The poplar industry clusters is formed by small micro-enterprises, this enterprise composition characteristic has positive influence on local urbanization. It is easy to found wood processing enterprises and engage in wood transportation and sale. Small businesses are flexible and employers can make full use of spare time and idle labors, so that their income is generally higher than migrant workers. Taking wood veneer manufacturing enterprises as an example, to establish a wood veneer business with three labor forces and annual output of $2000 \mathrm{~m}^{2}$, it needs to purchase a wood peeling machine, construct a shed and flat land 0.5 $\mathrm{hm}^{2}$, plus the purchase of raw materials, the total investment funds less than 50,000 RMB. In small or micro enterprises, employers usually work together with the employees. Employers can use the spare time before and after work to 
strip skin of logs, repair the veneer, maintenance the machine and do veneer product transactions. Besides, the women and the elderly at home can also work in the business. And the labor time of the workers is also flexible, they can determine the time of work and the length of the working hours according to the actual situation of individuals and enterprises. The flexibility of production and management leads to a higher level of income for both employers and employees than migrant workers. The enterprise composition characteristics of poplar industry cluster in northern Jiangsu can produce relatively high incomes, and then induce young and middle-aged laborers to work in local areas, thus becoming an important driving force of local population urbanization.

\subsection{The Influence Mechanism of Spatial Differentiation Characteristics of Polar Industrial Clusters on Local Urbanization}

Poplar industrial clusters in northern Jiangsu are spatially differentiated into point-like wood processing areas and surface-shaped poplar cultivation areas, which has a positive significance on the population of urbanization. Point-like wood processing areas have become the population attract center and have changed the characteristics of regional population flow. The development of the cluster led to the original rural settlements of wood processing areas evolution into town settlements. Spatially differentiated wood processing areas effectively shorten the distance between employment space and living place of the young and middle-aged labor forces, and it effectively reduce the phenomenon of young married labors to live alone, and then reduce the number of left-behind women, left-behind children and empty nest elderly people in rural areas, it helps to improve the quality of urbanization.

\section{Conclusions and Future Research Direction}

Through the analysis of the characteristics of poplar industrial cluster in Northern Jiangsu and their impact on population urbanization, we draw the following results and the future research direction.

\subsection{Conclusions}

The transformation of economic structure is the basis of urbanization in rural areas. The influence of the development of poplar industrial cluster on the urbanization of regional population shows that in the process of urbanization, the transformation of the vast rural areas from the traditional rural society to the urban society involves a series of changes in the economic structure, population structure, geographical space and way of life, in which the transformation of economic structure plays a decisive role and is the premise and basis of other changes. Therefore, the study of related issues about urbanization in specific areas should be based on regional economic structure and its changing characteristics. 
Characteristics of industrial clusters influence on the characteristics of urbanization. The characteristics of spatial indication and spatial differentiation of industrial clusters determine the regional characteristics of urbanization, and the characteristics of industrial structure and enterprises composition of industrial clusters determine the demographic characteristics of urbanization. Therefore, in the process of urbanization, different sizes of cities, towns or rural communities should develop different industrial clusters according to local economic and social conditions. For large cities, they should do their best efforts to develop technology intensive or intellectual intensive industrial clusters, to alleviate the contradiction between the number of people and the carrying capacity of resources in the process of urbanization. For small and medium-sized cities or rural communities may develop labor intensive industrial clusters, in order to play their population agglomeration effect in the process of urbanization.

The development of specific industrial clusters provides a material basis for local urbanization. In order to ensure the successful implementation of the local urbanization strategy, the rural areas should give full use their respective regional advantages and cultivate industrial clusters suitable for local natural, economic or social conditions, and provide material conditions for rural urbanization. The so-called local urbanization that lack of appropriate industry support is ineffective method, such as blindly encourage migrant workers to return home to do business and ask farmers to work in the local are not effective strategies.

\subsection{Future Research Direction}

This paper analyzes the changes of the characteristics of population flow, demographic structure characteristics and the settlement characteristics of the local urbanization from the perspective of industrial structure change caused by the industrial cluster development, but it does not consider the changes of the way of life of residential population in the process of local urbanization. This is the lack of this research and the author will improve it in the future.

\section{Acknowledgements}

This research was partly supported by the project of philosophy and social science research in the colleges and universities in Jiangsu province (No. 2016SJB840022).

\section{References}

[1] Zhu, Y. (2004) Changing Urbanization Processes and in Situ Rural-Urban Transformation: Reflections on China's Settlement Definitions, Hugo G. New Form of Urbanization, Aldershot, 207-228.

[2] Li, Q., Zhang, Y. and Chen, Z.H. (2016) Research on Models of Local Urbanization. Journal of Jiangsu Administration Institute, 1, 52-60.

[3] Jiao, X.Y. (2015) The Plights, Emphasis and Countermeasures in Situ Urbanization of New Urbanization Process: Another Idea of Controlling the "Urban Problems". Urban Development Studies, 22, 108-115. 
[4] Fei, X.T. (1984) Big Problem in Small Town. Outlook Weekly, 3, 24-25.

[5] Fei, X.T. (2000) Urbanization and Rural Development in China in Twenty-First Century. China Urban Economy, 1, 7-9.

[6] Lu, H., Y.C., Wang, H.G., et al. (2014) “In Situ Urbanization” Mode Co-Drive by Agriculture and Services: A Case of Dunhuang City, Gansu Province. Areal Researcher and Development, 33, 160-164.

[7] Yang, C.K. (2016) Population Agglomeration and Dynamic Mechanism of China's Multi-Scale Urbanization: From the Perspective of Population Mobility. PhD Thesis, East China Normal University, Shanghai.

[8] Yi, C. and Chen, Y.J. (2014) Research on the Mode of Rural Urbanization in "Post Crisis Era". Inquiry into Economic Issues, 1, 122-126.

[9] Qi, X.H., Zhu, Y. and Zhou, Y.P. (2012) A “Double-Pull” Model of Rural Labor Migration and Its in Situ Urbanization Effect: Cases Studies of Three Coastal Areas in Southeast China. Scientia Geographica Sinica, 32, 25-30.

[10] Patrick, M. (1991) Tourism Urbanization. International Journal of Urban and Regional Research, 3, 326-342.

[11] David, L.G. (1998) Tourism Urbanization in the United States. Urban Affairs Review, 1, 3-27.

[12] Zhu, Y. (2000) In Situ Urbanization in Rural China: Case Studies from Fujian Province. Development and Change, 31, 413-434. https://doi.org/10.1111/1467-7660.00160

[13] Pan, Y.H., Sun, Q., Meng, W.J., et al. (2014) In Situ Urbanization in Southwestern Mountainous Region. Planners, 4, 101-107.

[14] Porter, M.E. (1998) Clusters and New Economics of Competition. Harvard Business Review, Boston.

[15] Wei, S.H., Wang, J.C. and Zhao, Y.Q. (2002) Industrial Cluster: A New Theory of Regional Economic Development. Economic Survey, 2, 18-21.

[16] Wang, Z.Z. and Wang, F.X. (2005) Rethinking on the Puzzledom of the Growth-Pole Theory and Industrial Cluster Strategy. Human Geography, 6, 34-37.

[17] Shen, Z.P., Liu, H.J. and Jiang, T. (2004) On Relationship between Industrial Cluster and Regional Economic Development. China Soft Science, 2, 120-124.

[18] Wu, X.J. (2003) On the Significance of Industrial Cluster to the Development of Developing Areas. Jiangxi Social Sciences, 8, 123-126.

[19] Chen, L.S. (2005) Research on Promoting Regional Innovation System Construction with Industrial Cluster. Economic Survey, 4, 108-110.

[20] Huang, X.Z. and Cao, X. (2006) Industrial Clusters and Regional Innovation Capability. Economic Survey, 12, 31-37.

[21] Yang, D.P. (2004) On Industrial Clusters and Regional International Competitiveness. China Business and Market, 5, 41-44.

[22] Hu, B., Zhou, J.X. and Weng, Q.X. (2009) Study on the Effect of Hi-Tech Industry Clusters' Characteristics on Talents Attractiveness. R\&D Management, 2, 51-57.

[23] Li, B. and Li, L. (2014) Analysis on the Development of County Industrial Clusters and the Effect of the Knowledge Spillover in College's Talents Cultivation. Agricultural Economy, 8, 49-50.

[24] Duan, Q.Q. and Hou, G.M. (2011) Cultural Evolution and Industrial Cluster's Development. China Soft Science, S1, 176-179. 
[25] Yan, S.Q. (2015) Study on Characteristics and the Population Effects of the Polar Industrial Cluster in the North of Jiangsu. Journal of Coastal Development, 1 , 185-197.

[26] Yan, S.Q. (2015) Study on the Impact of the Industrial Cluster Characteristics on the Risks of Senior Citizens Life. Journal of Inner Mongolia Normal University (Philosophy and Social Sciences Edition), 44, 67-72. 